8

\title{
TOKAMAK FUSION TEST REACTOR (TFTR) NEUTRAL \\ BEAM LINE VACUUM CHAMBER COVER \\ STRUCTURAL ANALYSIS
}

D. L. Humphrey

L. R. Pedrotti

September 10, 1979

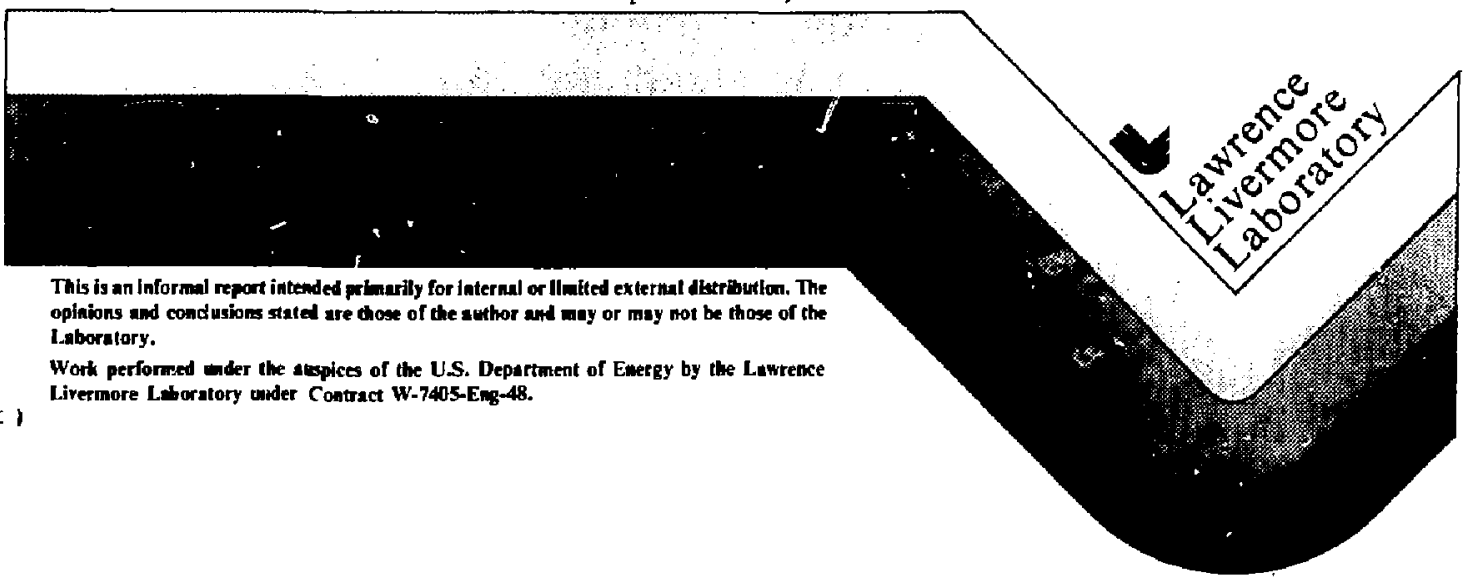

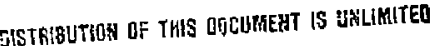




\section{TORAMAR FUSION TEST REACTOR (TFTR) NEUTRAL BEAM LINE VACUUM CHAYBER COVER STRUCTURAL ANALYSIS}

D. L. Humphrey and L. R. Pedrotri

Lawrence Livermore Laboratory, University of California Liveritare, GA 94550

\section{ABSTRACT}

The Tokamak Fusion Test Reactor (TFTR) vacuum chamber cover is sealed by 0-rings without the aici of mechanical fasteners. Under vacuum lo: ing and component wights, the edges lifted no more than 0.005 in. (by a SAP4 computer code analysis). This report explains the model used for this investigation and, in addition, shows the maximum deflection expected at the center of the cover is less than 0.047 in. Also, no stresses are expect: to exceed $13,700 \mathrm{psi}$.

\section{INTRODUCTION}

Most components of the neutral beam 1 ines of the Tokamak Fusion Test Reactor (TFTR) are enclosed in a large, right-parallel-piped vacuuth chamber. The function and rationale for the unorthodox design of this structure are explained in the referenced publication.*

₹[. C. Pittenger, et aI., "Neutral Beam Injection System for the Tokamak Fusion Test Reactor," Proceedings of the 7th Symposium on Engineering Problers in Fusion Kesearch, Oct. 1977 Conference, p. 555.

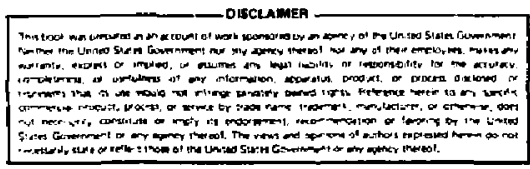


One unusual feature of this chamber is a cover that sits on seals without being mechanically fastened to the lower section of the chamber. Under pressure and other structural loads, the cover tends to lift around the edges. While the seals are designed to tolerate some lift around the edges, we started this investigation to more accurately evaluate seal integrity for several loading conditions. Useful by-products of the analysis are the stresses and bending moments in the structural members.

TOP COVER DESCRIPTION

As shown in Fig. 1, the cover is a reinforced flat plate made of AISI 300 series stainless steel with two large access ports and several stiffeners, For convenience, we affixed a set of coordinate axes with the origin at point lying at half the width of the panel, the left end of the panel, and in the plane of the bottom panel face. The plate thickness was originally planned to be $0.75 \mathrm{in}$. but has been changed to 2 in. The access ports are 0.75-in.-thick plate with 2-in.-thick covers. The intersections of the access ports and bottom plate are reinforced by 2.5-in.-thick stiffening rings.

Other reinforcements consist of the fabricated box beam around the perimeter and fabricated T-beams traversing the plate. The box beam, which has cross-sectional dimensions of $9 \mathrm{in}$. wide by $12 \mathrm{in.} \mathrm{high,} \mathrm{is} \mathrm{built} \mathrm{from}$ 0.75-in.-thick plate. The two transverse T-beams are $12 \mathrm{in.} \mathrm{high} \mathrm{and} \mathrm{have}$ 0.75-in. webs and 1.5-in. by 6-in. flanges. The short longitudinal T-beams are also 12 in, high with $0.75-i n$. webs and $0.75-i n$. by 6-in. Elanges.

Besides supforting the pressure load and ics own gravity load, the top panel supports several other components. A calorimeter, weighing $8000 \mathrm{lbf}$, is suspended from the center of the cover of the smaller access port. The 
ion dump, weighing $1076 \mathrm{lbf}$, is suspended from the large access port cover in two places. A liquid helium Dewar flask sits on the longitudinal T-beams and weighs $3000 \mathrm{lbf}$. A cryopanel, weighing approximately $6120 \mathrm{lbf}$, hangs from the top panel along each side of the chamber. Each panel is suspended from four points with the line of suspension about $40 \mathrm{in}$. from the centerline of the top panel.

MODELING ASSUMPTIONS AND COMPUTATIONAL PROCEDURE

THE MESH

We used the finite element method to approximate the deflections and rotations in a static, linear elastic analysis. Symetry about the $y-z$ plane allows us to model only half the structure by impressing the proper boundary conditions on nodes in the plane of symetry. We modeled the structure as 417 thin plate elements joined at 424 nodal points, as shown in Fig. 2. This geonetry and associated boundary conditions are input to the SAP4 computer code, which computes the approximate derlection? and rotations at each nodal point. The resulting matrix system involves 2441 degrees of freedom.

Due to the size of the grid and limitations on available software, the mesh was generated in several sections and merged by a text editing program. This disjoint generation sequence yielded element orderings resulting in a half-bandwidth of 1848 . The size of this matrix system required more contiguous disk storage space than is usually available. Preprocessing of the element connectivity data by the SAPlis computer code reduced the half-bandwidth to 152 . This results in much more efficient 
$$
-4-
$$

computations due to fewer disk information transfers required for matrix formulation and equation elimination.
\end{abstract}

BOUNDARY CONDITIONS AND LOAD CASES

Symmetry about a plane allows us to reduce the problew size by half with apecial consideration for the boundary conditions on noges in the plane of symmetry. Consider a plane bisecting a beam perpendicular to the longitudinal axis of the beam. Asoume the beam is simply supported and :oaded symmetrically about the bisecting plane. For small displacements, points in this plane have no axial displacement, and the plane undergoes no rotation. Analogous boundary conditions are impressed on this structural problem. For all lcad cases considered later, Dodes in the plane of symmetry are constrained to zero displacement perpendicular to the plane, and no rotation of the plane is permitted.

The first computations in the analysis yield a solution for the case of only the component and gravity loads discussed earlier without the pressure luad. This simulates no-vacuum conditions and determines seal integrity at the beginning of chamber evacuation. The second computation simulates normal operating conditions with gravity loads and a pressure load of $16 \mathrm{lbf} / \mathrm{in}^{2}$. This pressure load is excessive as a safety factor. While we are interested in displacements and stresses throughout the panel, behavior around the outer edge is of cencral importance. The contact problem between the top panel and the sides of the chamber is nonlinear due to constraint in one direction (one surface contacts another) and no constraint in another (one surface loses contact with another). We attack the problem with an iterative procedure gimilar to a computational procedure employed in contact problems. Springs are used to constrain all 
displacements normal to the contact surface. This problem is solved, and the extension of each spring is considered. If spring is compressed, indicating the surfaces are in contact, the spring is not removed. However, if a spring is extended, tending to pull two uncontacted surfaces together, the spring is removed, and the computation is repeated. This iterative process is continued until all remaining springs are compressed, and inclusion of any other springs would result in an extended spring. Then the contact surface is defined, and displacements of uncontacted nodes may be used to evaluate seal integrity.

\section{SPRING STIFFNESSES}

A satisfactory model for this problem might include the cover and portions of the sides and ends of the chamber that contact the top. However, this problem would be prohibitively large, so we attempt to model the effect of the remainder of the chan'jer on the cover through the springs around the panel's perimeter.

The problem arises as to what value of spring rate will adequately reflect the effect of the sides on the cop. We approach this problem by approximating a spring rate based on uniaxial compression of the sides by the top. In the first serieg of computations, we set the spring rates to a much higher value to establish an upper bound on the edge displacements. Later, we solve the problem with the derived spring rates. We compare the results of the ce computations in evaluating seal integrity.

Considering a uniaxial tension test, we see that

$$
F=\frac{A E}{\bar{L}} \delta,
$$


where

F - Force in the member.

A - Cross-sectional area of the nember.

E - Young's Modulus.

L - Length of the member.

$\delta$ - Change in length $L$ oue to load F.

Then $K=A E / L$ is the spring constanL for the member. From the design drawings, we choose the following figures to desctibe the side and end panels:

$\begin{array}{lllcc} & \begin{array}{l}\text { Width } \\ \text { (in.) }\end{array} & \begin{array}{l}\text { Length } \\ \text { (in.) }\end{array} & \begin{array}{c}\text { Area } \\ \text { (in. })\end{array} & \begin{array}{c}K \\ \text { (1bf/in.) }\end{array} \\ \text { Side panels: } & 198.0 & 154.25 & 234.9 \text { in.* } & 4.1 \times 10^{7} \\ \text { End panels: } & 118.0 & 164.0 & 88.6 \mathrm{in.} & 1.5 \times 10^{7}\end{array}$

ॠIncludes area of T-beams.

For the upper bound on spring rates we choose $10^{10} \mathrm{lbf} / \mathrm{in}$.

It is clear that better models may be developed for the spring rates along the edges. For example, the spring rates near a corner should be much larger than those in the midsection. However, we choose to base our recomendations on the two rates given here, as the original question concerns seal integrity and not precise edge deflections.

COMPUTATIONAL RESULTS

For this first series of computations, we attempt to establish upper bovads on edge deflections by assuming boundary spring rates of $10^{10} \mathrm{lbf} / \mathrm{in}$. Results of interest may be best expressed by referring to a 
plot of the finite element mesh of the bottom plate only. This plot, with some selected nodal point numbers, is shown in Fig. 3 . We consider first the case of structural loads but no pressure load.

After several spring removal iterations, the contact surface is defined by nodes 1-3, 38-165, and 190-192. The maximum deflection around the perimeter of the top panel is 1.44 mils at node 7 . These results indicate that the seal will be intact when no pressure load exists. We next consider the case of structural losds plus the pressure load to simulate fully evacuated conditions. The resulting contact surface is defined by nodes 1-4, 33-157, and 190-193. The maximum vertical edge deflection is 5.4 mils at node 197. Again the seal should remain intact. We also find that the maximum vertical deflection of the panel is $-48.1 \mathrm{mils}$ at node 44. Maximu principal stresses throughout the structure are less than 13,500 pei.

Considering the conservative nature of the spring rate assumption for the preceding calculations and the indications that the seal will be effective, we omit computations for the $10^{7} \mathrm{lb} / \mathrm{in}$. spring rate. 


$$
-8-
$$

\section{FIGURE GAPTIONS}

FIG. 1. Perspective view of the chamber top panel.

FIf 2. Three-dimensional perspective computer plots of the finite element mesh for the top panel.

FIG. 3. Relevant nodal point numbers on the lower surface of the top panel.

$\mathrm{JD} / \mathrm{mr}$ 


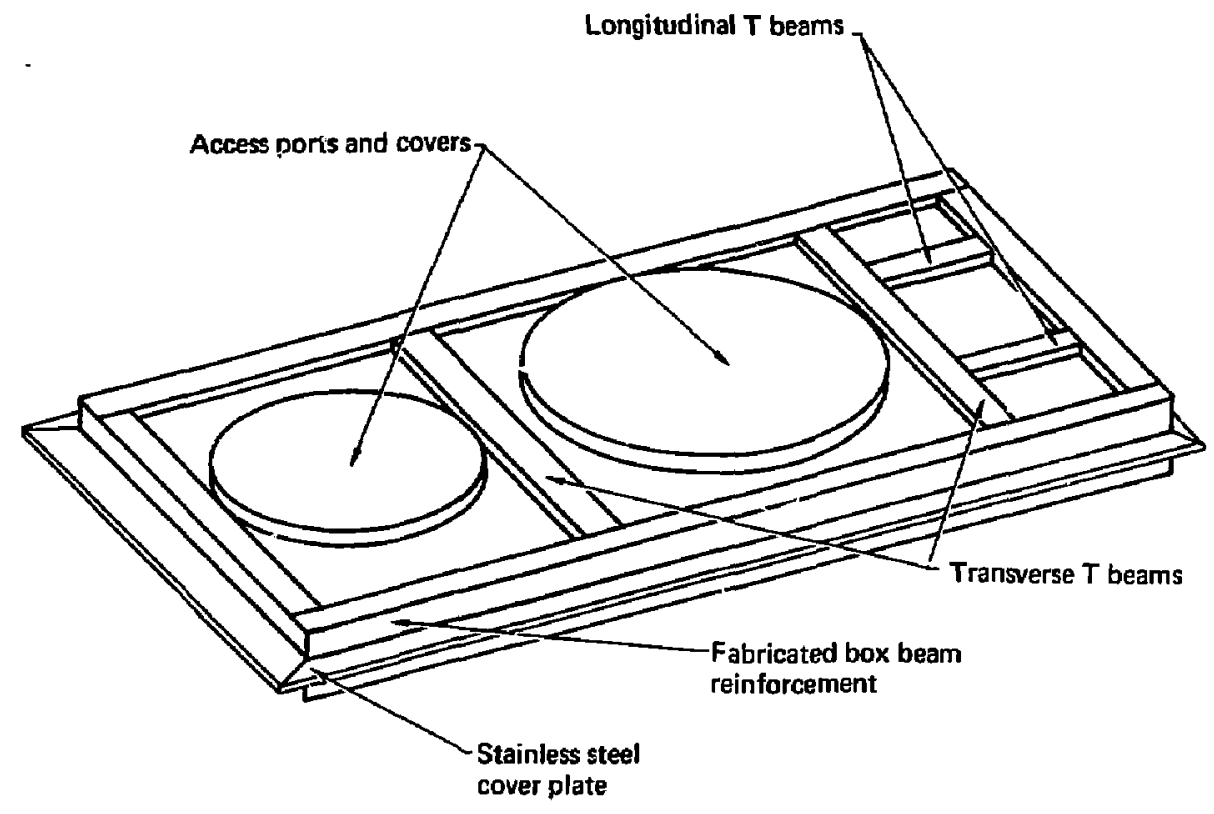

F1gure 1. 

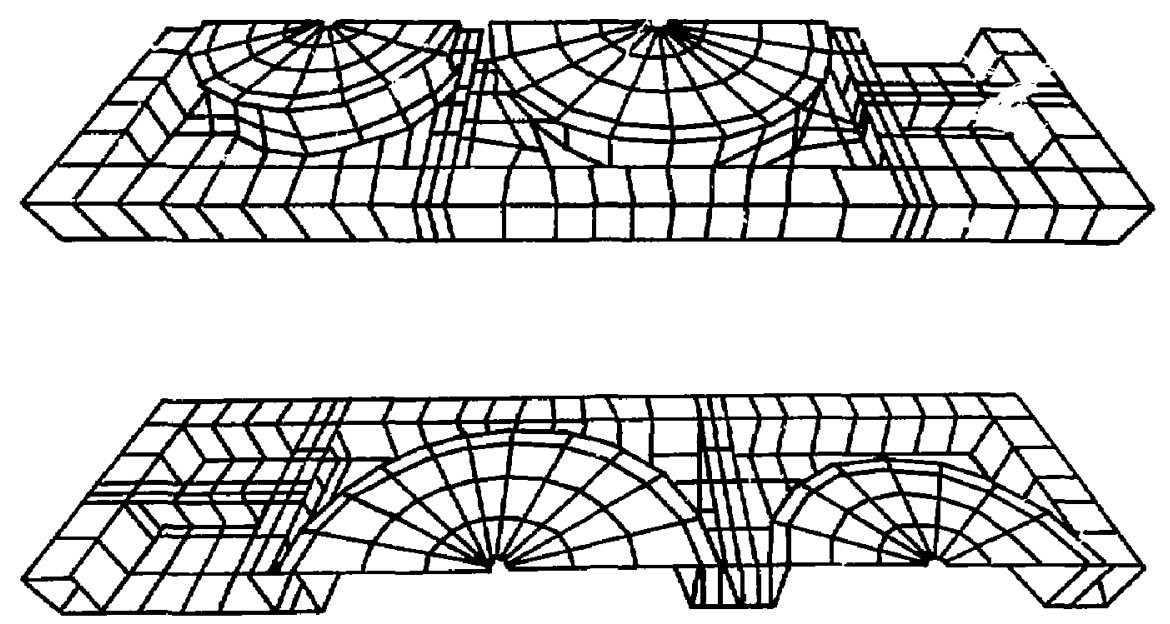

Figure 2. 


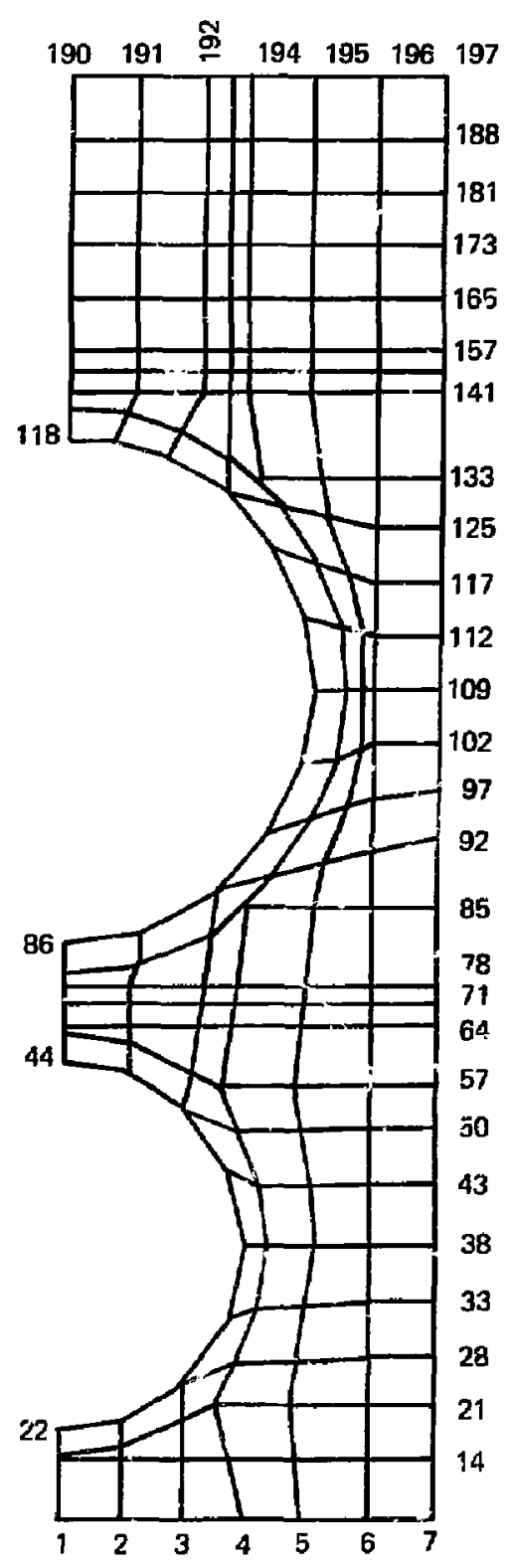

Figure 3. 